\title{
Online Advertisement Service Pricing and an Option Contract
}

\author{
Yongma Moon ${ }^{\mathrm{a}}$, Changhyun Kwon ${ }^{\mathrm{b}, *}$ \\ ${ }^{a}$ Department of Industrial and Manufacturing Engineering, the Pennsylvania State \\ University, yongma@psu.edu \\ ${ }^{b}$ Department of Industrial and Systems Engineering, University at Buffalo, SUNY, \\ chkwon@buffalo.edu
}

\begin{abstract}
For the Internet advertisement market, we consider a contract problem between advertisers and publishers. Among several ways of pricing online advertisements, the methods based on cost-per-impression (CPM) and costper-click $(\mathrm{CPC})$ are the two most popular. The $\mathrm{CPC}$ fee is proportional to the click-through rate (CTR), which is uncertain and makes decisions of advertisers and publishers difficult. In this paper, we suggest a hybrid pricing scheme: advertisers pay the minimum of CPM and CPC fees by purchasing an option from publishers. To determine the option price, we consider a Nash bargaining game for negotiation between an advertiser and a publisher and provide the solution. Further, we show that such option contracts will help the advertiser avoid high cost and the publisher generate more revenue. The option contract will also improve the contract feasibility, compared to CPM and CPC.
\end{abstract}

Keywords: online advertisements, option contract, Nash bargaining, click-through rate, utility maximization

\footnotetext{
${ }^{*}$ Corresponding author. Tel: +1-716-645-4705, Fax: +1-716-645-3302
} 


\section{Introduction}

Over the last decade, the Internet has emerged as an important medium for advertising. According to a recent report of the Interactive Advertising Bureau (2008), over $\$ 23$ billion has been spent in the U.S. market alone. For the first 14 years of Internet advertising history after it first was introduced in 1995, the market increased by more than $400 \%$ in terms of total revenue. In 2008, the Internet advertising market was the third largest in the U.S. after only television channels (including national, local and cable stations) and newspapers. The American Press Institute (2009) reported that the Internet advertising market took only 13 years to reach $\$ 20$ billion in revenue, while newspapers took 127 years in the U.S. Without doubt, the Internet is the fastest growing marketing medium in history.

The Interactive Advertising Bureau (2008) also reported that searchbased keyword advertising achieved $45 \%$ in revenue share in 2008 , while the second on the list was display banner advertising with a $20 \%$ revenue share. Retailers and financial service providers spent $22 \%$ and $13 \%$ of the total revenue, respectively, which were the top two expenditures in the market.

Although the marketing media have been growing very fast, there is still much confusion over how advertisement publishers (e.g., web content providers) should charge for and how advertisers (e.g., retailers) should pay for their advertisement campaigns. When the market was first introduced, the traditional cost-per-impression method was used. It charges a fixed cost for a given number of displays of banner ads. As the biggest online advertisers are retailers, the actual benefit of advertisement campaigns usually comes from visits of customers to and purchases from the website of advertisers. In such cases, the number of displays does not reflect the benefit of advertising. Many advertisers believe that it is not reasonable to pay for advertisements that generate no value.

As the number of clicks likely reflects advertising effects, later in the history of the Internet marketing, payment based on the number of clicks has become very popular. This method is called cost-per-click, or CPC, while the traditional method based on the number of display is often called cost-per-mille, or CPM (mille is a Latin word meaning thousand). The $\mathrm{CPC}$ is possible, because the exact number of clicks is trackable due to the nature of information technology (Hoffman and Novak 2000). The CPC is one of several performance-based payment methods. Among other such methods, the cost-per-action, or CPA method is popular. This is based 
on the number of observable actions, for example, purchases, subscriptions, membership registrations, etc. In 2008, CPC pricing and other performancebased pricing made up 57\% of the total revenue, while CPM pricing made up $39 \%$. The CPC pricing is particularly popular in search-based advertising, while the CPM pricing is popular in banner display advertising. The market for CPA pricing is not yet mature, because the number of actions is neither controllable nor tractable by the publisher.

In CPC contracts, the click-through rate (CTR), which is the ratio of the number of clicks to the number of displays, plays an important role. The advertiser receives more marginal benefit from advertising when the CTR is higher. Although in CPC contracts the advertiser pays an advertising fee based on the number of clicks, which potentially reflects the actual advertising benefit, there is a significant drawback in CPC pricing. Click-frauds, which do not lead to purchases or subscriptions, will increase the CTR drastically without contributing to the profit of the advertiser. Many CPC contract providing publishers and agencies maintain click-fraud detection systems, but there is no perfect detection technology. Even without considering click-frauds, the CTR is never known a priori, hence both publishers and advertisers will be uncertain about it. Lahaie et al. (2007) noted that CTR can fluctuate dramatically even over small periods of time. In practice, if the same advertisement has been displayed by the same publisher, the historical estimate of CTR is used, while a forecast is used when the advertisement is completely new.

As we noted earlier, different types of contract schemes have been invented to generate more revenues or extract more advertisers' interests from the publisher's perspective. However, among those contracts, an advertiser has to select only one of them. As mentioned, since one contract can be more beneficial than the others, the contract choice might be crucial to the publisher's revenue. For example, suppose that an advertiser selects a CPC contract before she advertises when she is uncertain about the CTR. After its ads appear, if the CTR turns out to be high, then the advertisers might wish she had contracted to use CPM instead, since this will cost less.

In this paper, we propose a risk management method to deal with the uncertainty about the CTR and analyze the potential of this method to increase the likelihood of contract agreement. As a way to hedge against risk, we consider an instrument for the minimum price guarantee for the advertiser, with which she can choose between the CPM and CPC pricing after the CTR is realized. Of course the advertiser will choose the minimum 
of the CPM and CPC fees. To earn this privilege, the advertiser must pay an additional fee. Obviously if the additional fee is too high, the advertiser will not consider making the contract with the minimum price guarantee. The additional fee will be determined by a negotiation between the advertiser and the publisher. We call this contract type with the minimum price guarantee an option contract and call the additional fee for the guarantee the option price, following the theory of financial options (Wilmott et al. 1993). To determine the option price, we use an approach that is risk-neutral to both contract parties based on our analysis of a Nash bargaining game (Nash 1950). Furthermore, we investigate the potential of the option contract as a new hybrid pricing scheme. We make an assumption that the advertisement effect depends only on the number of clicks, which is not restrictive when we consider that the largest group of advertisers is retailers. In summary, the research objective of this paper is to propose a novel contract, option, and then investigate the potential of the contract.

The paper is organized as follows. In Section 2, we review the literature regarding online advertisements involving CPM and CPC contracts. We also discuss option contracts that have been suggested in other service industries. We define the option contract and formulate the option pricing model with the other two popular pricing contracts in Section 3. We show that the option contract has a better possibility of contract agreement compared to the standalone CPM and CPC contracts in Section 4. We provide managerial insights from the option contract in Section 5, and study the problem numerically in Section 6. We conclude in Section 7.

\section{Literature Review}

With the radical growth of the Internet, researchers have been giving new attention to the theoretical problems associated with online advertising. For instance, they have studied how to optimally schedule advertisements with limited resources (Adler et al. 2002; Dawande et al. 2003; Amiri and Menon 2006). These papers presented a way to maximize revenue of a web site owner from advertising by determining the optimal sequencing of advertisements. Another stream of research analyzes bidding mechanisms and finds equilibria in search engine auctions (Aggarwal et al. 2007; Feng et al. 2007; Varian 2007; Liu et al. in press). For example, Athey and Ellison (2009) built models considering consumer search behavior and then analyzed the resulting equilibria for the design of sponsored keyword auctions. 
However, relatively few studies regarding different contract types have been reported in the literature. Mangani (2004) addressed optimal decision making between CPM and CPC advertisements when the publishers are price-takers in the display advertisement market and later Fjell (2009) revisited the same problem. Both articles consider a revenue maximization problem with a deterministic CTR. Kwon (in press) also studied a capacity allocation problem between CPM and CPC advertisements with stochastic page-view and CTR. Kumar and Sethi (2009) considered a dynamic pricing problem considering subscription and advertising and Roels and Fridgeirsdottir (2009) studied a dynamic optimal customer selection and display scheduling problem considering only CPM contracts. Fridgeirsdottir and Najafi-Asadolahi (2009a,b) also studied revenue management problems with CPM contracts. In contrast to these approaches that were developed for existing pricing schemes, we propose a novel option contract in this paper.

Our option contract, as noted earlier, has a similar concept to financial options, in particular, European call options (Wilmott et al. 1993). With a European call, the option holder can purchase an asset with the minimum of the exercise price and the current market price, after the asset price is realized at the expiration date. For the option contract to be agreed on, the option holder should pay a certain amount of money, namely the option price, in advance. Options are widely used and traded in financial markets by investors who want to hedge their risk. The analogy behind these financial options is very similar to the option contract proposed in this paper in the sense that an option holder has flexibility in contract selection. However, unlike the asset price in a European option, we do not assume that CTR follows a dynamic stochastic process.

Utilization of option products or contracts, as suggested in our paper, has been highlighted as a good marketing or operational strategy in some other service industries. For example, Gallego and Phillips (2004) suggested the use of options thinking in the airline industry. They viewed the airline company as having a right to assign customers who purchase a flexible option product to take another flight at the end of a given period of time. Then, they showed that the flexible products have the advantage of increasing overall demand and using capacity more efficiently. Similarly, Gallego et al. (2008) proposed a callable service product which can be used for capacity expansion when full fare demand exceeds available capacity. Levin et al. (2007) introduced an option model to support a price assurance policy in service revenue management area to extract demand from the market. 
There are other studies that apply real option theory to IS or e-commerce area (Campbell 2002; Kumar 2004), but they are a little different from our paper in the sense that the real option theory is used to evaluate an investment. To our knowledge, no attention has been paid to an option contract for online advertisement, even though it can be used as a good tool to hedge risk. To fill this gap, we propose an option contract and analyze its potential. Our model is based on game theoretic approach.

\section{Model Setting and Option Value}

In our model, we focus on two parties involved in an online advertising contract: an online advertiser and an online advertisement publisher. An advertiser wants to market products (or services) to consumers by delivering online advertisements to consumers who may be interested. On the other hand, a publisher wants to make additional revenue by selling small online "real estate" with high page-views which may attract advertisers' attention. Usually, publishers own original content web sites, for example, world news, specialized information, current affairs, information technology trends, market surveys, etc.

We consider only display advertisements such as web banners, where the identities of advertisers and publishers are unambiguous. For example, if an advertiser wants to display web banners at the New York Times website, the publisher is obviously the New York Times. However, in advertising service systems like Google AdSense, advertisers do not know where their advertisements will be displayed, and publishers do not know whose advertisements will be displayed at their web sites. Service systems determine which advertisements to display based on "relevance" of banners to participating web sites. The publisher-advertiser matching algorithm is dependent on how the advertising service systems define "relevance". As we will consider negotiations between advertisers and publishers, such advertising service systems will not be of interest to us. We also do not consider the search-based keyword advertising market like Google Adwords, Yahoo Search Marketing, Microsoft adCenter, etc. In the search advertising market, CPC pricing is usually used and it is prominently based on auctions for higher CPC. 
Throughout this paper, we use the following notation:

$$
\begin{aligned}
& n: \text { number of impressions } \\
& p: \text { cost per impression, or CPM } \\
& \pi: \text { cost per click, or CPC } \\
& C: \text { click-through rate, or CTR (random variable) } \\
& V: \text { option value, or option price }
\end{aligned}
$$

An impression is defined as an instance of the advertisement being displayed in a visitor's browser. The number of impressions may be controlled by a publisher in some sense. For instance, a publisher can stop exposing an advertisement after a certain number of impressions, or can control the sequence of advertisements shown in the web page to make the contracted number of impressions realized. However, it is uncertain how many customers are interested in the advertiser's products. Needless to say, so is the number of clicks by those customers.

For display advertising markets, we suggest an option contract as follows. Suppose there are two viable pricing schemes already, CPM and CPC which are prevalently used in the market. We assume the publisher already has a novel method to determine $p$ and $\pi$. Now, the publisher proposes an option contract. To make the option contract, the advertiser should pay option price $V$ to the publisher in advance. Later, when the contract is over and the advertiser pays the advertisement fee, the advertiser has a right (or an option) to choose between CPM and CPC.

Let us discuss how the option contract is constructed and is used in our problem. The advertisement fees for two methods are defined as:

$$
\begin{array}{r}
p n: \text { CPM fee } \\
\pi C n: \text { CPC fee }
\end{array}
$$

where $C n$ is the number of clicks and $C$ is the random CTR. By an option contract, the advertiser can have an option to choose the best payment mode, that is, $\min (p n, \pi C n)$. In other words, by paying additional $V$, the advertiser can protect herself from uncertainty. Suppose the CTR is very high so that $p n<\pi C n$. With CPC pricing, the advertiser has to pay $\pi C n$ regretting she should have contracted for CPM pricing. Now, suppose the CTR is very low so that $p n>\pi C n$. With CPM pricing, even though the advertisement received fewer clicks (or attentions), the advertiser has to pay $p n$ because 
the advertisement was simply displayed $n$ times. Because the real effect of advertisement comes from clicks in most cases, this could be problematic to the advertiser. However, the option contract enables the advertiser to avoid these worst cases. On the other hand, the publisher can have an opportunity of making additional profits by collecting the option price $V$.

Let us now model the utility of advertiser for the option contract. The utility of the option contract is

$$
Q \equiv E[-V-\min (p n, \pi C n)+a \pi C n]
$$

where $V+\min (p n, \pi C n)$ represents the total advertising cost and $a \pi C n$ is the marketing effect gained by $C n$ number of clicks with the proportional coefficient $a \pi$ with a constant $a>0$. We define the marketing effect as a measure of how much benefit the advertiser would gain by advertising. With this utility model, we assume the marketing effect of online advertising is only proportional to the number of clicks. Although there have been debates about how to effectively measure the marketing effects of online advertisements, the number of clicks is still one of the most important measure of these effects.

Similarly, for the publisher, we can write the profit in agreement with the option contract as follows:

$$
P \equiv E[V+\min (p n, \pi C n)-b p n]
$$

where $V+\min (p n, \pi C n)$ is the total revenue and bpn represents the cost to display $n$ times with the coefficient $b p$ with a constant $b>0$. The cost to display web banners includes the opportunity cost by losing a chance of hosting network advertisements, which are advertisements offered and maintained by agencies or ad networks. Hosting network advertisements provides guaranteed chances of CPM advertisements at lower revenue, but without the promised number of displays responsible (Roels and Fridgeirsdottir 2009). This means there is no reason for the publisher to make an option contract, if the revenue from the option contract is less than the revenue from the risk-free network advertisements.

However, we know that these expected utilities are obtained, only when both parties agree to the option contract. When two individuals have the opportunity to collaborate for mutual benefit, they encounter a cooperative bargaining situation (Nash 1950). Through collaboration, both can obtain mutual profit and negotiate a division of that profit. However, if both parties fail to reach an agreement, this mutual benefit could be lost. In our 
problem, if a publisher and an advertiser succeed in agreeing to a contract, both parties can be better off. An advertiser can also make a profit by selling more products (marketing effect) and will pay a portion of the profit to a publisher as an advertising cost. Also, we know that the share for each party is dependent on the negotiation outcome (option value $V$ ) and neither party can get anything if the option is not contracted. Thus, this paper uses the cooperative bargaining model to find an optimal allocation of the profit (optimal option value $V$ ) between a publisher and an advertiser.

Furthermore, in a bilateral negotiation, it is reasonable to expect that the player with higher negotiation power (the more powerful player) will have a larger share than the weaker player. Here, the negotiation power means the ability of each party to influence the opponent's action and it is relative between parties. For example, usually advertisers like Target or eTrade have higher power than publishers, because advertisements are the main source of income to online publishers in most cases, while online advertisements are optional to the advertisers. Also, such high volume advertisers will likely continue to advertise with the same publishers who give better terms to the advertisers. However, higher power publishers such as ESPN.com and the New York Times would be in a position superior to some advertisers, because there are many alternative advertisers who want to display their banners on the web sites with higher attention. To incorporate the negotiation power, the axioms by Nash (1950) can be extended to the Generalized Nash Bargaining (GNB) game as discussed in Roth (1979) and Nagarajan and Sošić (2008). The generalized Nash solution, $B\left(x_{1}, x_{2} ; d_{1}, d_{2} ; \alpha, \beta\right)$, is obtained by solving the following form (Nagarajan and Sošić 2008):

$$
B\left(x_{1}, x_{2} ; d_{1}, d_{2} ; \alpha, \beta\right)=\arg \max \left(x_{1}-d_{1}\right)^{\alpha}\left(x_{2}-d_{2}\right)^{\beta}
$$

where the constants $\alpha$ and $\beta$ describe the relative negotiation powers and $\alpha+\beta=1$. If player 1 is more powerful in the negotiation than player 2 , then $\alpha$ is larger than $\beta$. When an agreement is reached, the players will have utilities of $x_{1}$ and $x_{2}$. Also, $d_{1}$ and $d_{2}$ are the disutilities when the parties fail to reach an agreement. These disutilities are usually assumed to be zero, because neither would have anything to lose or gain if the negotiation fails.

By applying the theory of Generalized Nash Bargaining games to our problem, we can determine the contract price $(V)$. To reach an agreement, both parties will bargain for the contract price of the option with their relative negotiation powers. The parties can expect utilities like (1) and (2), when the 
option contract is agreed. Negotiating the contract condition for the option value $V$, both parties will reach an equilibrium. Thus, the option value at the equilibrium is derived by GNB as follows:

$$
V=\arg \max \Pi \equiv \arg \max Q^{\gamma} P^{1-\gamma}
$$

where $\gamma$ is the relative negotiation power of the advertiser. Based on the bargaining game, we can determine the option price as in Theorem 1 . We assume that the advertiser's and publisher's utilities are nonnegative; otherwise, both parties will not accept this contract and the contract will be infeasible. We will discuss contract feasibility later.

Theorem 1 (Option Value for Internet Advertising Contracting). Option value determined by the Nash bargaining game (3) is

$$
\begin{aligned}
V & =-E[\min (p n, \pi C n)]+(1-\gamma) a \pi n E[C]+\gamma b p n \\
& =a \pi n E[C]+\gamma(b p n-a \pi n E[C])-n \pi \int_{0}^{p / \pi} c f(c) d c-n p\left(1-F\left(\frac{p}{\pi}\right)\right)
\end{aligned}
$$

where $f(\cdot)$ and $F(\cdot)$ are the probability density function and the cumulative distribution function of the CTR, $C$, respectively.

Proof. We will find an optimum $V$ maximizing $\Pi$, in terms of the first order condition and the second order condition. However, since solving directly is not easy because of powers in $\Pi$, we will take a logarithm of $\Pi$. We have

$$
\begin{aligned}
& \log \Pi=\gamma \log (-V-E(\min (p n, \pi C n))+a \pi n E[C]) \\
&+(1-\gamma) \log (V+E(\min (p n, \pi C n))-b p n)
\end{aligned}
$$

From the first order condition, we have

$$
\begin{aligned}
& \frac{\partial}{\partial V} \log \Pi \\
& =\frac{-\gamma}{-V-E(\min (p n, \pi C n))+a \pi n E[C]}+\frac{1-\gamma}{V+E(\min (p n, \pi C n))-b p n} \\
& =0
\end{aligned}
$$

or

$\gamma[V+E(\min (p n, \pi C n))-b p n]=(1-\gamma)[-V-E(\min (p n, \pi C n))+a \pi n E[c]]$ 
The option value $V$ becomes

$$
\begin{aligned}
V & =-E[\min (p n, \pi C n)]+(1-\gamma) a \pi n E[C]+\gamma b p n \\
& =-E[\min (p n, \pi C n)]+a \pi n E[C]+\gamma(b p n-a \pi n E[C])
\end{aligned}
$$

We know that

$$
\begin{aligned}
E[\min (p n, \pi C n)] & =n E[\min (p, \pi C)] \\
& =n \pi \int_{0}^{p / \pi} c f(c) d c+n p \int_{p / \pi}^{\infty} f(c) d c \\
& =n \pi \int_{0}^{p / \pi} c f(c) d c+n p\left(1-F\left(\frac{p}{\pi}\right)\right)
\end{aligned}
$$

Therefore, the option value at equilibrium is

$$
V=a \pi n E[C]+\gamma(b p n-a \pi n E[C])-n \pi \int_{0}^{p / \pi} c f(c) d c-n p\left(1-F\left(\frac{p}{\pi}\right)\right)
$$

We check the second-order derivative:

$$
\begin{aligned}
& \frac{\partial^{2}}{\partial V^{2}} \log \Pi \\
& =\frac{-\gamma}{\{-V-E(\min (p n, \pi C n))+a \pi n E[C]\}^{2}}+\frac{-(1-\gamma)}{\{V+E(\min (p n, \pi C n))-b p n\}^{2}} \\
& <0
\end{aligned}
$$

for all $\gamma \in(0,1)$. Hence, the proof is complete.

The option value is affected by the negotiation power of the advertiser and the publisher. Next, we will examine the relationship between option value and negotiation power in the following theorem.

Theorem 2 (Advertiser's and Publisher's Negotiating Power). The option value is decreasing in the advertiser's negotiation power and increasing in the publisher's negotiation power. That is,

$$
\frac{\partial V}{\partial \gamma} \leq 0, \quad \frac{\partial V}{\partial(1-\gamma)} \geq 0
$$


Proof. For an option contract to be feasible, we should have

$$
\begin{aligned}
& Q=E[-V-\min (p n, \pi C n)+a \pi C n] \geq 0 \\
& P=E[V+\min (p n, \pi C n)-b p n] \geq 0
\end{aligned}
$$

Therefore, we should have

$$
b p n \leq E[V+\min (p n, \pi C n)] \leq a \pi n E[C]
$$

From the first order derivative of $V$ with respect to negotiation power $\gamma$, we obtain

$$
\frac{\partial V}{\partial \gamma}=b p n-a \pi n E[C] \leq 0
$$

Similarly, we have

$$
\frac{d V}{d(1-\gamma)}=a \pi n E[C]-b p n \geq 0
$$

which completes the proof.

Theorem 2 explains that an advertiser can agree to the contract with a lower option value, when an advertiser holds a superior position in a negotiation.

\section{Contract Feasibility}

In this section, we investigate contract feasibility. We compare the option contract with standalone contracts based on CPM and CPC. In the CPM contract, the advertiser's utility is

$$
Q_{C P M}=E[-p n+a \pi C n]
$$

and the publisher's utility is

$$
P_{C P M}=E[p n-b p n]
$$

In the same manner, the utility functions of advertiser and publisher, for the CPC contract, are

$$
\begin{aligned}
Q_{C P C} & =E[-\pi C n+a \pi C n] \\
P_{C P C} & =E[\pi C n-b p n]
\end{aligned}
$$


respectively.

However, the option contract may not be contractible because of its unprofitability to either the advertiser or the publisher. For example, if the expected utility of the advertiser with the option contract is negative, $Q<0$, the advertiser will not consider the option contract. This is also true for the publisher: she will not consider it when $P<0$. Thus, we discuss when the option contract becomes feasible and compare the feasibility to other contracts. Let $\bar{C}$ denote the minimum expected value of the CTR that makes the option contract feasible and $\bar{C}_{C P M}$ and $\bar{C}_{C P C}$ for the CPM and CPC contracts, respectively. That is,

$$
\begin{aligned}
\bar{C} & \equiv \inf \{E[C]: \text { the option contract is feasible }\} \\
\bar{C}_{C P M} & \equiv \inf \{E[C]: \text { the CPM contract is feasible }\} \\
\bar{C}_{C P C} & \equiv \inf \{E[C]: \text { the CPC contract is feasible }\}
\end{aligned}
$$

In the subsequent lemmas, we derive $\bar{C}, \bar{C}_{C P M}$ and $\bar{C}_{C P C}$.

Lemma 1 (Option Contract Feasibility). The condition for the option contract being feasible is:

$$
\bar{C}=\frac{b p}{a \pi}
$$

Proof. For feasibility, we must have

$$
\begin{aligned}
& Q=E[-V-\min (p n, \pi C n)+a \pi C n] \geq 0 \\
& P=E[V+\min (p n, \pi C n)-b p n] \geq 0
\end{aligned}
$$

From Theorem 1, we know

$$
V=-E[\min (p n, \pi C n)]+(1-\gamma) a \pi n E[C]+\gamma b p n
$$

Substituting (6) to (4) and (5), we obtain the conditions for feasibility:

$$
\begin{aligned}
& Q=\gamma[a \pi n E[C]-b p n] \geq 0 \\
& P=(1-\gamma)[a \pi n E[C]-b p n] \geq 0
\end{aligned}
$$

From (7) and (8), we have threshold $\bar{C}$

$$
E[C] \geq \frac{b p}{a \pi} \equiv \bar{C}
$$


Unless the condition given in Lemma 1 is satisfied, both parties would not consider the option contract. In other words, a too low level of CTR could make the option contract less attractive to both parties. In the following lemmas, we will investigate similar conditions so as to compare CPM and CPC contracts to the option contract.

Lemma 2 (CPM Contract Feasibility). The conditions for the CPM contract being feasible are:

$$
\bar{C}_{C P M}=\frac{p}{a \pi}, \quad b \leq 1
$$

Proof. The feasibility conditions for the CPM contract are

$$
\begin{aligned}
Q_{C P M} & =-p n+a \pi E[C] n \geq 0 \\
P_{C P M} & =p n-b p n \geq 0
\end{aligned}
$$

which lead to

$$
E[C] \geq \frac{p}{a \pi} \equiv \bar{C}_{C P M}, \quad b \leq 1
$$

For the CPM contract, the publisher's utility does not depend on the CTR, while the advertiser's does. We note that if the CTR is too low, the CPM contract is less attractive to the advertiser, because the advertiser may have to pay more than the actual advertisement effect.

Lemma 3 (CPC Contract Feasibility). The conditions for the CPC contract being feasible are:

$$
\bar{C}_{C P C}=\frac{b p}{\pi}, \quad a \geq 1
$$

Proof. The feasibility conditions for the CPC contract are

$$
\begin{aligned}
Q_{C P C} & =E[-\pi C n+a \pi C n] \geq 0 \\
P_{C P C} & =E[\pi C n-b p n] \geq 0
\end{aligned}
$$

which lead to

$$
a \geq 1, \quad E[C] \geq \frac{b p}{\pi} \equiv \bar{C}_{C P C}
$$


The CPC contract is less attractive to the publisher, since she could collect less revenue than the actual cost to display, when the CTR is less. On the other hand, the advertiser pays proportional to the actual benefit from advertising.

Now, we present the following important result:

Theorem 3 (Option Contract Feasibility Comparison). From the perspective of contract feasibility, the option contract is more feasible than the $C P M$ and $C P C$. In particular

$$
\bar{C} \leq \bar{C}_{C P M} \quad \text { and } \quad \bar{C} \leq \bar{C}_{C P C}
$$

Proof. Let us compare $\bar{C}$ and $\bar{C}_{C P M}$. We have

$$
\bar{C}_{C P M}-\bar{C}=\frac{(1-b) p}{a \pi} \geq 0
$$

since $b \leq 1$ for the feasibility of the CPM contract. Therefore, $\bar{C}_{C P M} \geq \bar{C}$. Similarly we have

$$
\bar{C}_{C P C}-\bar{C}=\frac{p(a-1) b}{a \pi} \geq 0
$$

since $a \geq 1$ for the feasibility of the CPC contract. Therefore $\bar{C}_{C P C} \geq \bar{C}$ and this completes the proof.

For a low CTR, when the CPM or CPC standalone contracts are less attractive to the advertiser and publisher, the option contract can be feasible. We note that this better contract feasibility of the option contract does not necessarily guarantee higher utility for both parties. The better feasibility implies that the possibility of negotiation agreement becomes higher, because both parties can agree even for a lower CTR. From the publisher's perspective, the better feasibility will potentially attract more advertisers to the publisher. Suppose some possible advertisers in the market are worrying about low CTR which might incur losses. If a publisher offers only CPC or CPM, a low CTR could be critical. However, since an option contract requires a lower threshold $\mathrm{CTR}$, the advertisers and the publisher who have hesitated making a contract with CPM and CPC might consider contracts with the option. Hence the option contract will potentially provide more revenue to the publisher, who will, in turn, enjoy bigger market share.

Obviously, the option contract is beneficial to the advertiser. For example, when the CTR is expected to be very low, the advertiser would like 
to make a CPC contract but the publisher probably does not want to contract. Thus, this situation may lead to contract disagreement. However, the option contract enables the publisher to consider accepting the contract, because the option price provides additional compensation to the publisher. Also, the advantage of the option contract can be found in the sense of riskhedging. The advertiser can protect herself from higher cost if the CTR is too high. A high CTR, which leads to a high CPC cost, may come from click frauds. Most publishers maintain automated click frauds detection systems, but such detection would be very difficult to be perfect. When the cost of advertising is too high due to a high CTR, the advertiser will pay no more than the CPM cost, which does not increase with the CTR.

\section{Managerial Insights}

Even though we have shown that utilization of the proposed option contract can improve contract feasibility, how the option contract could work in a real world and why it is necessary in a practical sense might be unclear. Thus, further investigation about the option is discussed in this section (i) from the qualitative viewpoint (regret) and (ii) quantitative viewpoint (utility). Let us consider a case in which both parties have contracted with an option with price $V$ and they now realize a CTR after a contract period. The reader should notice that while $V$ is determined by the probabilistic distribution information of the CTR, $C$, the realized utility depends on the realization of the CTR. Let $\tilde{c}$ denote a realization of the CTR. Then for a given $\tilde{c}$, the realized utility of the advertiser for each contract is as follows:

$$
\begin{aligned}
\tilde{Q}(\tilde{c}) & =-V-\min (p n, \pi \tilde{c} n)+a \pi \tilde{c} n \\
\tilde{Q}_{C P M}(\tilde{c}) & =-p n+a \pi \tilde{c} n \\
\tilde{Q}_{C P C}(\tilde{c}) & =-\pi \tilde{c} n+a \pi \tilde{c} n
\end{aligned}
$$

Similarly, the realized utility of the publisher for each contract is as follows:

$$
\begin{aligned}
\tilde{P}(\tilde{c}) & =V+\min (p n, \pi \tilde{c} n)-b p n \\
\tilde{P}_{C P M}(\tilde{c}) & =p n-b p n \\
\tilde{P}_{C P C}(\tilde{c}) & =\pi \tilde{c} n-b p n
\end{aligned}
$$


We compare the realized utilities and regrets of the advertiser and the publisher. The regret is the difference between the maximum utility that each part could have achieved and the realized demand. For example:

Advertiser's Regret for the Option Contract $(\tilde{c})$

$$
=\max \left(\tilde{Q}(\tilde{c}), \tilde{Q}_{C P M}(\tilde{c}), \tilde{Q}_{C P C}(\tilde{c})\right)-\tilde{Q}(\tilde{c})
$$

The regret for a publisher is defined similarly.

Based on the utility functions and regret functions, we have the following interesting results.

Theorem 4 (Publisher's Maximum Profit and Advertiser's Constant Regret). Publishers can generate the maximum profit with an option contract when

$$
|p n-\pi \tilde{c} n|<V
$$

where $\tilde{c}$ is the realized CTR. In other words, $\tilde{P}(\tilde{c}) \geq \max \left(\tilde{P}_{C P M}(\tilde{c}), \tilde{P}_{C P C}(\tilde{c})\right)$. In addition, advertisers can expect a stable level of regret regardless of the realized CTR. In particular

$$
\text { Advertiser's Regret for the Option Contract }(\tilde{c})=V \quad \text { (constant) }
$$

Proof. To examine the relationship between $\tilde{P}(\tilde{c})$ and $\mathrm{V}$, we observe

$$
\begin{aligned}
& \tilde{P}(\tilde{c})-\max \left(\tilde{P}_{C P M}(\tilde{c}), \tilde{P}_{C P C}(\tilde{c})\right) \\
& =V+\min (p n, \pi \tilde{c} n)-b p n-\max \left(\tilde{P}_{C P M}(\tilde{c}), \tilde{P}_{C P C}(\tilde{c})\right) \\
& =V+\min (p n, \pi \tilde{c} n)-b p n-\max (p n, \pi \tilde{c} n)+b p n \\
& =V+\min (p n, \pi \tilde{c} n)-\max (p n, \pi \tilde{c} n)=V-|p n-\pi \tilde{c} n|
\end{aligned}
$$

Thus, if $|p n-\pi \tilde{c} n|<V$, then the option contract is the most beneficial.

Regret for an advertiser is defined as:

$$
\begin{aligned}
\max \left(\tilde{Q}(\tilde{c}), \tilde{Q}_{C P M}(\tilde{c}), \tilde{Q}_{C P C}(\tilde{c})\right)-\tilde{Q}(\tilde{c}) \\
=\max (-V-\min (p n, \pi \tilde{c} n)+a \pi \tilde{c} n, a \pi \tilde{c} n-\min (p n, \pi \tilde{c} n)) \\
\quad+V+\min (p n, \pi \tilde{c} n)-a \pi \tilde{c} n \\
=a \pi \tilde{c} n-\min (p n, \pi \tilde{c} n)+V+\min (p n, \pi \tilde{c} n)-a \pi \tilde{c} n \\
=V \text { (constant) }
\end{aligned}
$$

Therefore, we can see that regret is not affected by the realized CTR. 
The above results show that an option contract can be beneficial to both parties. To help the reader to understand our results in Theorem 4, we provide a numerical example and an explanation. Let us consider a case in which the parameters and the option value are determined by the following: $a=2, b=0.1, p=0.007, \pi=7, n=100,000$, and $V=224 .^{1}$

The realized utility and regret of advertiser for each contract are presented in Figures 1 and 2, respectively. We note that the advertiser's utility for the option contract is the least around $E[C]$, where the two other utility lines meet. However, when the CTR is very different than $E[C]$, the advertiser's utility of option contract is never the least. This concept is very similar to that of health insurance. If the insured person is never ill, the money for the health insurance policy is wasted. On the other hand, if she happens to become ill, and hence is exposed to high medical cost, she is protected by the insurer. In the option contract we propose, the advertiser is protected from high risk, but has to pay the option price. This becomes more obvious when we compare the regret. The advertiser's regret of option contract is always the option price she paid to the publisher. However, for other contracts, CPM and $\mathrm{CPC}$, the regret can be very high. With this protection, the advertiser can plan their marketing strategy and expenses in a more stable way with less variation. This protection is more useful, when a large CTR results from click-frauds, which increases the advertiser's cost without contributing to the benefit.

For the publisher, the opposite phenomenon occurs. Again, in Figure 3, $E[C]$ is the point where the utility lines of CPM and CPC contracts meet. If the realized CTR, $\tilde{c}$, is as close as the a priori expected value of CTR, $E[C]$, the publisher's utility is greatest. However, even when $\tilde{c}$ is far away from $E[C]$, the publisher's utility is not the least, because she is paid the option price. In Figure 4, the publisher's regret is presented for each contract. As we can easily see, the publisher's regret is relatively bigger for smaller and bigger CTR realizations, and is smaller, in fact, the least, for CTR realizations close to $E[C]$. Again, we can give an interpretation similar to the health insurance case. The insurer carefully determines insurance prices based on each insured person's background and information. If the insurer's forecast about each

\footnotetext{
${ }^{1}$ While we chose $a, b$ and $n$ are arbitrarily, we used the pricing strategy, $\$ 7$ per a thousand display, of ESPN.com (as of Jan 18, 2010) with the industry average CTR, $0.10 \%$ (DoubleClick 2009). The option value $V=224$ is computed using a gamma distribution.
} 


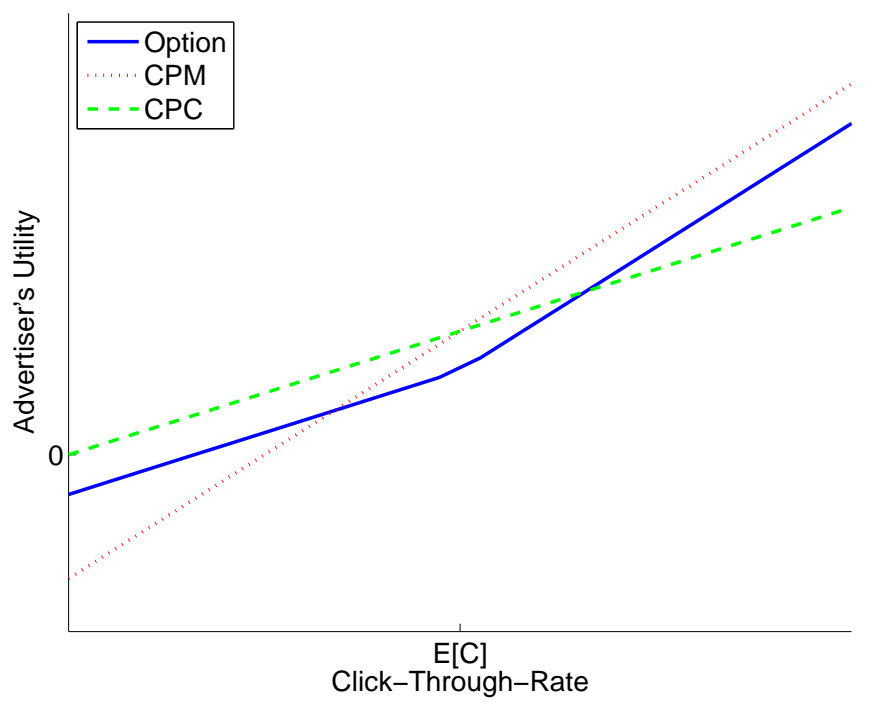

Figure 1: Comparison of advertiser's utility

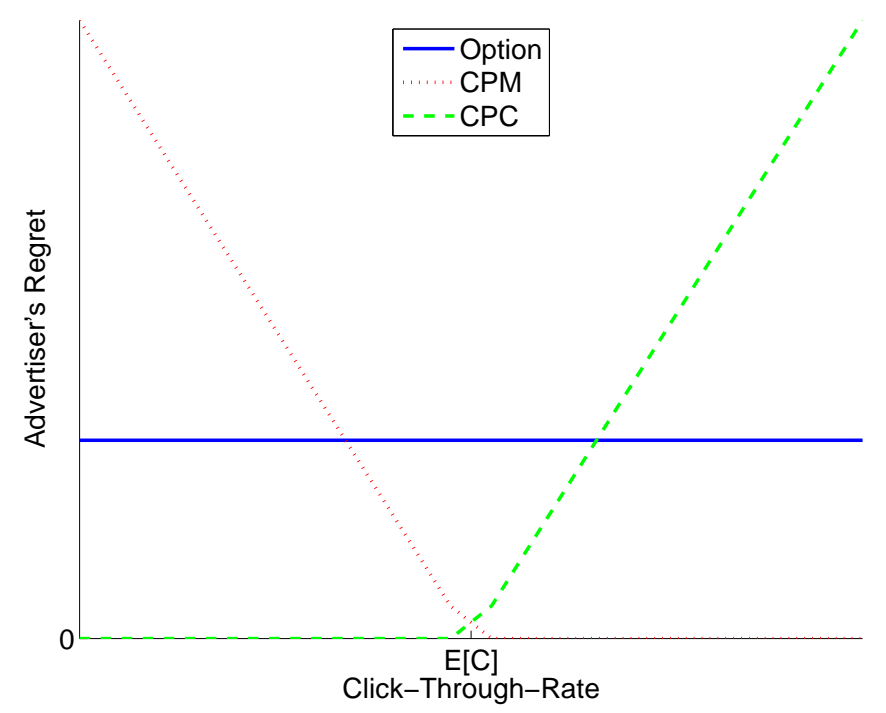

Figure 2: Comparison of advertiser's regret 


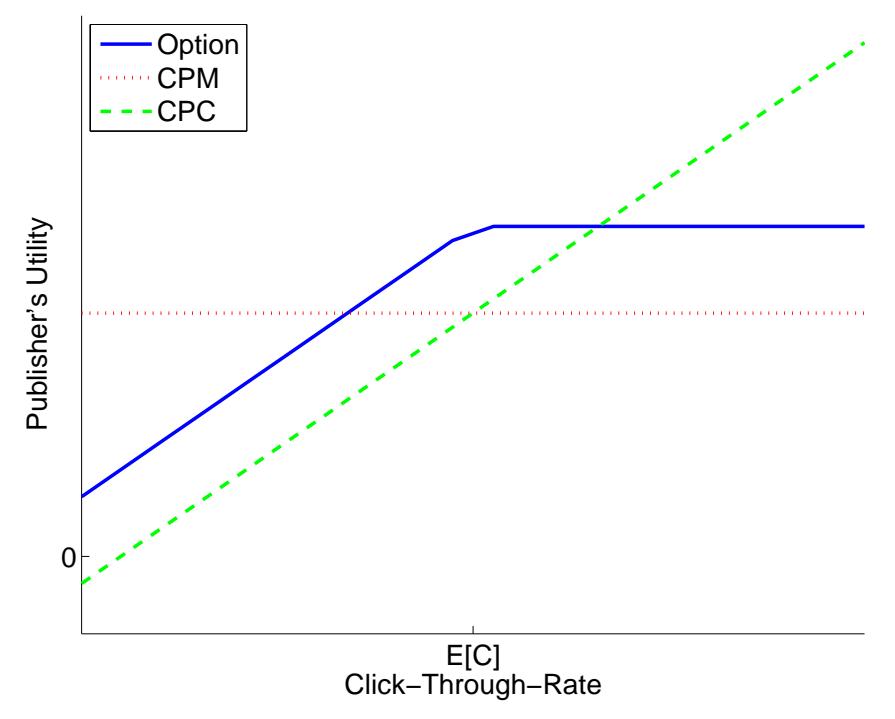

Figure 3: Comparison of publisher's utility

insured person's medical needs is incorrect, the insurer is responsible for high medical costs which are much higher than the insurance price. However, in most cases, the insurer's forecast is accurate enough. This is how insurance companies make a profit. In online advertisement markets, publishers usually have better information and forecasting about CTR, hence, publishers can generate more revenue by granting option contracts. In addition, as noted in Theorem 3, the option contract can be agreed to for a lower CTR. Therefore, the publisher can potentially attract more customers.

Next, let us examine condition (9). It can be written as $\left|\frac{p}{\pi}-\tilde{c}\right|<\frac{V}{\pi n}$. That is, the option contract will be beneficial to the publisher when the CTR is realized in the following interval:

$$
\frac{p}{\pi}-\frac{V}{\pi n}<\tilde{c}<\frac{p}{\pi}+\frac{V}{\pi n}
$$

Considering that often $p$ and $\pi$ are set to so as to make $p=\pi E[C]$, we conclude that the option contract brings additional revenue to the publisher, when the CTR is realized near the value the publisher forecasted. At the same time, the existence of this condition also implies that more accurate estimation of the CTR is very important to the publisher. In Figure 5, we 


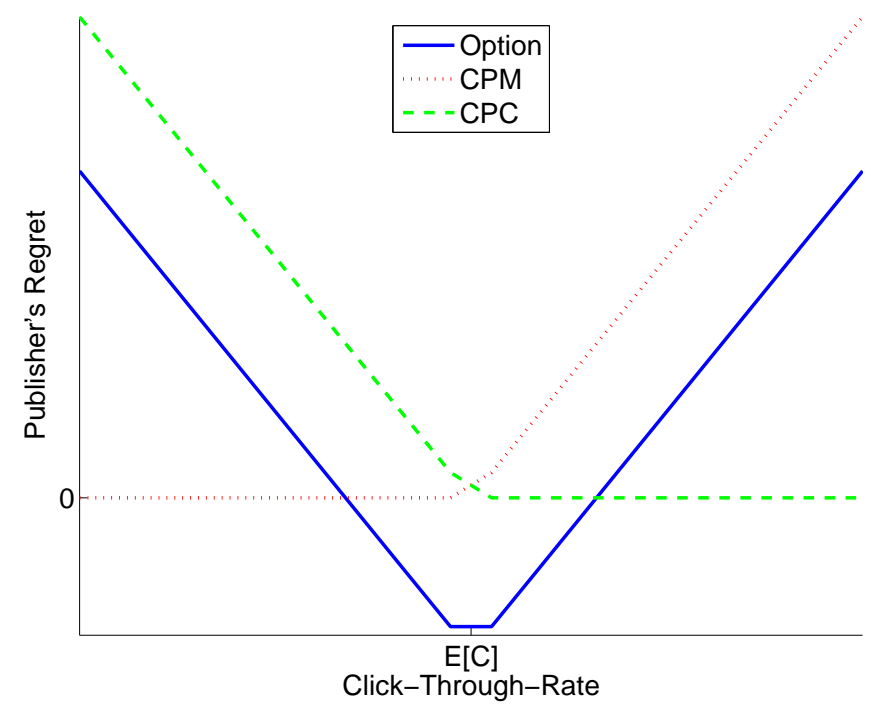

Figure 4: Comparison of publisher's regret

show the interval (10) to the axis of $p / \pi$, given $\pi=7$ fixed. We choose $p / \pi$ axis, since the ratio $p / \pi$ is meaningful when we compare it with the CTR. In Figure 5, for each $\pi$ and $p / \pi$, if the CTR is realized on the shaded area, the publisher generates additional revenue by the option contract.

\section{Numerical Examples}

In this section, we provide some numerical examples for different distributions of the CTR. One important question to address is the following: is the expected CTR pricing scheme, $p=\pi E[C]$, enough for the risk neutrality of both advertisers and publishers? If the option value becomes zero for the expected CTR pricing, we may conclude that the expected CTR pricing is just enough and there is no need to consider an option. However, we will see the option value will neither be zero nor be the smallest for the expected CTR pricing.

For asymmetric distribution cases, it is rather obvious that the option contract is useful when the expected CTR pricing scheme is used. However, based on the results of our numerical experiments, we will see that 


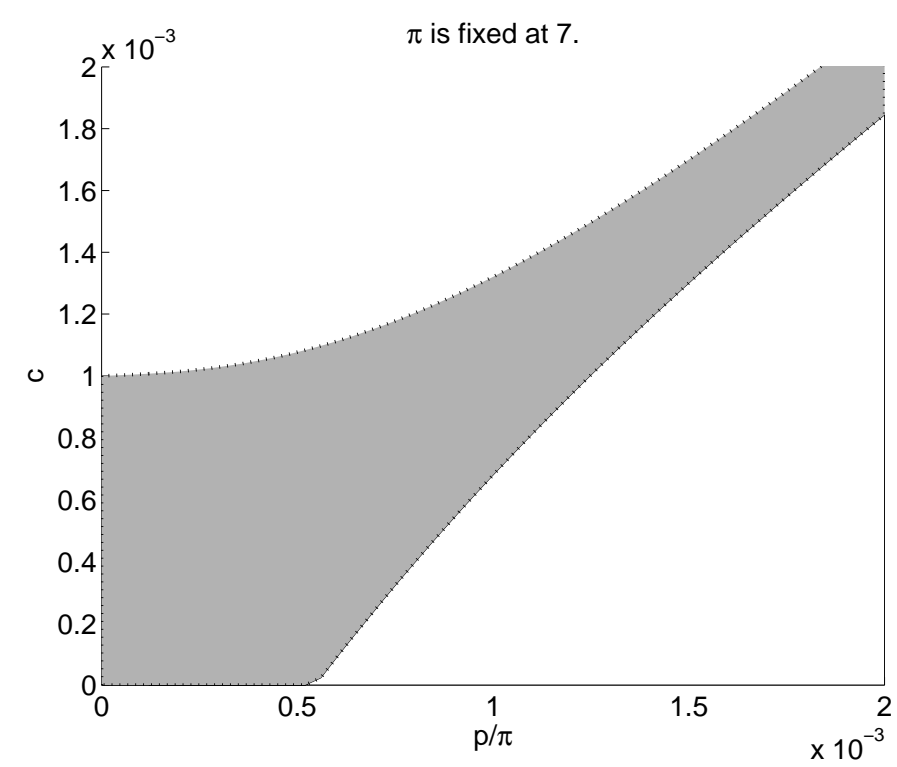

Figure 5: Boundaries of CTR where the option contract is profitable to the publisher when $\pi$ is fixed at $\$ 7$.

the expected CTR pricing scheme is not enough in both the symmetric and asymmetric distribution cases. ${ }^{2}$

First, we test an asymmetric gamma distribution case, and then we test a symmetric uniform distribution case. The numerical examples in this section are developed given that $a=1$ and $b=1$. Also, we assume both an advertiser and a publisher have the same negotiation power, so $\gamma=1 / 2$.

\subsection{Gamma Distribution Case}

Let us assume CTR follows a gamma distribution as follows:

$$
C \sim \operatorname{Gamma}(k, \theta)
$$

\footnotetext{
${ }^{2}$ However, Robinson et al. (2007) noted that CTR is skewed, empirically.
} 
with $k$ an integer, $\theta$ a constant and

$$
\begin{aligned}
E[C] & =k \theta \\
f_{k, \theta}(c) & =c^{k-1} \frac{\exp (-x / \theta)}{(k-1) ! \theta^{k}} \\
F_{k, \theta}(c) & =1-\sum_{i=0}^{k-1} \frac{(x / \theta)^{i}}{i !} \exp (-x / \theta)
\end{aligned}
$$

where $f_{k, \theta}(\cdot)$ and $F_{k, \theta}(\cdot)$ are the probability density function and the cumulative density function, respectively. Then, from the Theorem 1 , we have

$$
V=\frac{1}{2}(a \pi n k \theta+b p n)-n \pi k \theta F_{k+1, \theta}\left(\frac{p}{\pi}\right)-n p\left(1-F_{k, \theta}\left(\frac{p}{\pi}\right)\right)
$$

We use the following parameters:

$$
\begin{aligned}
& n=100,000, p=0.01, \pi=10 \\
& k=2, \theta=0.0005, E[C]=k \theta=0.001
\end{aligned}
$$

This set of parameters is chosen to make $p=\pi E[C]$. In this case, we obtain

$$
V=270.67
$$

while we have

$$
\begin{aligned}
E[\mathrm{CPM} \text { fee }] & =p n=1,000 \\
E[\mathrm{CPC} \text { fee }] & =\pi E[C]=1,000
\end{aligned}
$$

We have also computed the option value with different values of $p$ and $\pi$. In Figures 6 and 7, we present CPM cost, CPC cost and the associated option value with varying $p$ and $\pi$, respectively. As seen in those figures, the minimum option value does not occur at the point such that $p=\pi E[C]$ where the $\mathrm{CPM}$ and $\mathrm{CPC}$ cost lines intersect, and the minimum option value is not zero. This reflects not only the skewness of gamma distributions but also the risk induced by the stochasticity of the problem. In Figure 8, we provide a 3 -dimensional view of the option value with varying $p$ and $\pi$. 


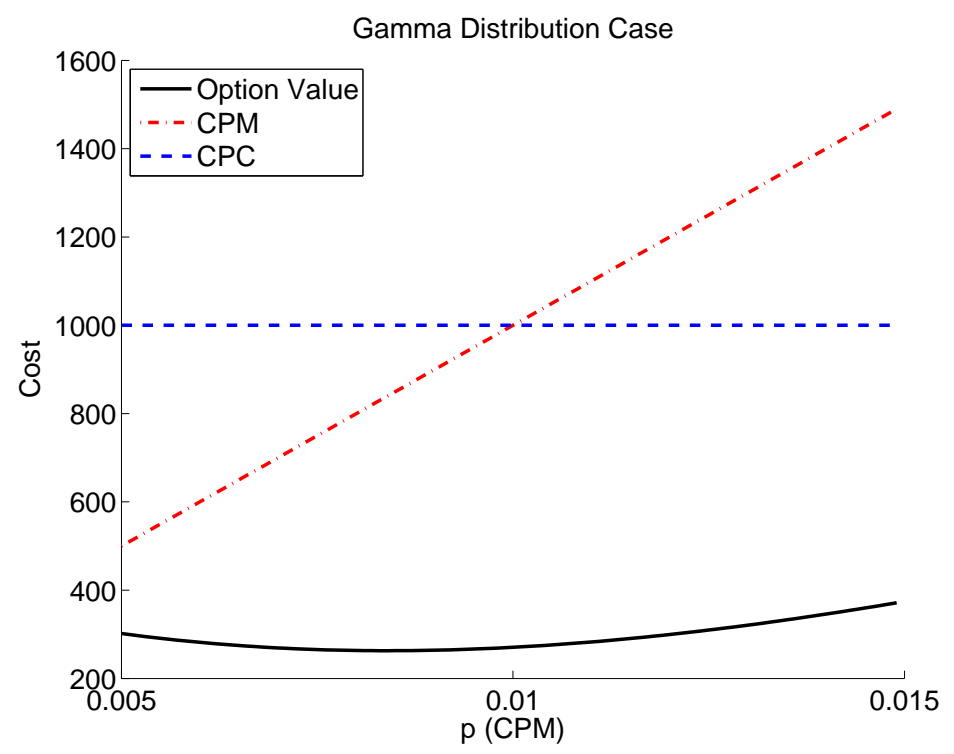

Figure 6: Option values with different $p$ (CPC)

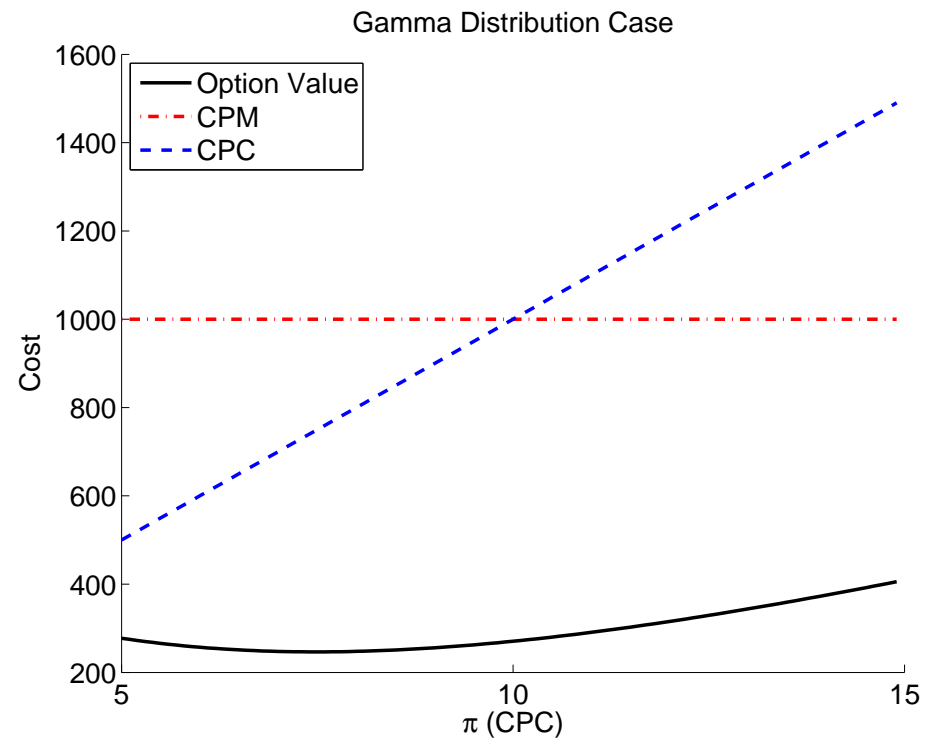

Figure 7: Option values with different $\pi$ (CPM) 


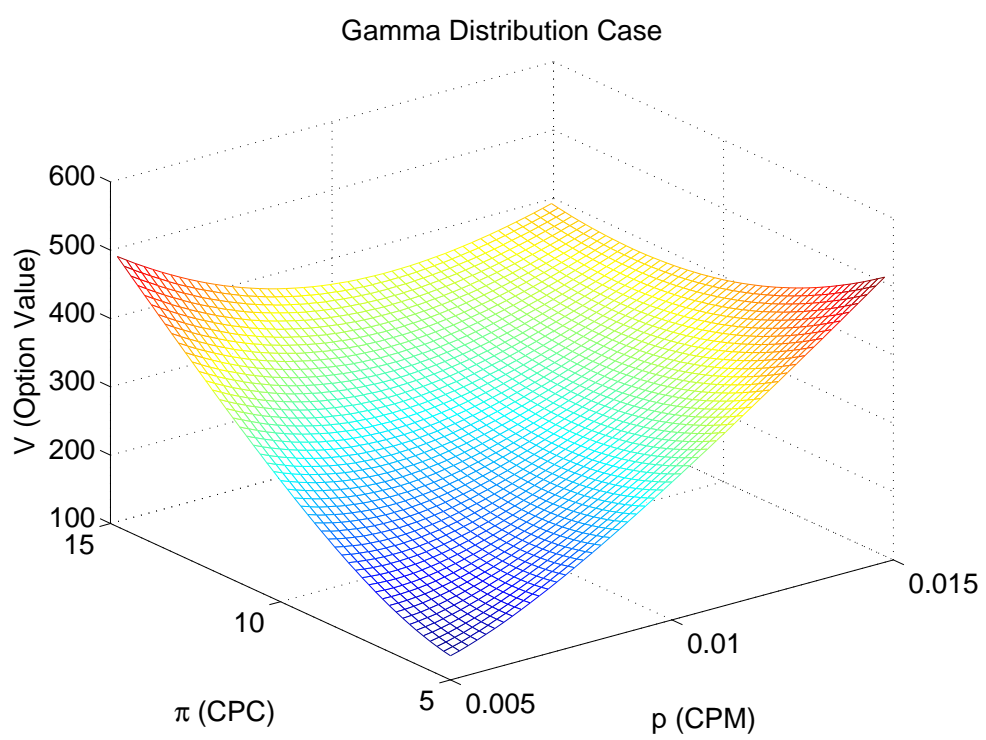

Figure 8: Option values with different $p(\mathrm{CPM})$ and $\pi(\mathrm{CPC})$

\subsection{Uniform Distribution Case}

Let us look at a symmetric distribution of the CTR. In particular, we consider a uniform distribution: $C \sim \operatorname{Uniform}\left(c_{1}, c_{2}\right)$. Then the option value is as follows:

$V=\frac{1}{2}\left(a \pi n \frac{c_{1}+c_{2}}{2}+b p n\right)-\frac{n \pi}{c_{2}-c_{1}}\left[\frac{(p / \pi)^{2}}{2}-\frac{\left(c_{1}\right)^{2}}{2}\right]-n p\left(1-\frac{p / \pi-c_{1}}{c_{2}-c_{1}}\right)$

for $c_{1} \leq p / \pi \leq c_{2}$;

$$
V=\frac{1}{2}\left(a \pi n \frac{c_{1}+c_{2}}{2}+b p n\right)-n p
$$

for $p / \pi<c_{1}$; and

$$
V=\frac{1}{2}\left(a \pi n \frac{c_{1}+c_{2}}{2}+b p n\right)-n \pi \frac{c_{1}+c_{2}}{2}
$$

for $p / \pi>c_{2}$. We choose $c_{1}=0.0003$ and $c_{2}=0.0017$. 


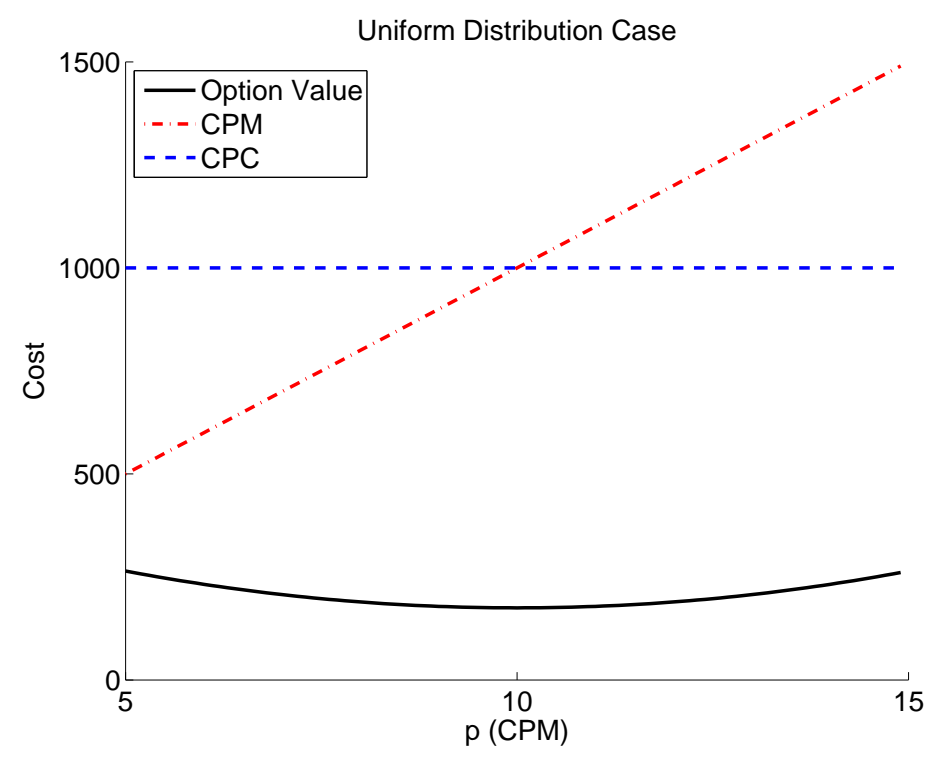

Figure 9: Option values with varying $p(\mathrm{CPM})$

We repeated the same experiments and present the results in Figures 9, 10 and 11. We note that, in Figure 10, the minimum option value does not occur at the intersection for 'symmetric' uniform distribution case.

If we do not consider a probabilistic distribution of $C$, and we use only the expected value $E[C]$, the $\mathrm{CPC}$ must be $\pi=p / E[C]$. However, even in the case of symmetric uniform distributions, we observe pricing based on the expected CTR is not enough, that is, $V \neq 0$ at the intersection in Figure 10.

\subsection{Variance of CTR and Option Value}

We further study the relation between variance of CTR and option value. For the uniform distribution, we consider the interval $\left[c_{1}, c_{2}\right]$ with the fixed mean $\bar{c}=\frac{c_{1}+c_{2}}{2}$. We let $c_{1}=\bar{c}-\delta$ and $c_{2}=\bar{c}+\delta$. Then the variance of the uniform distribution is obtained by

$$
\operatorname{Var}=\frac{1}{12}\left(c_{2}-c_{1}\right)^{2}=\frac{1}{12}(2 \delta)^{2}=\frac{1}{3} \delta^{2}
$$




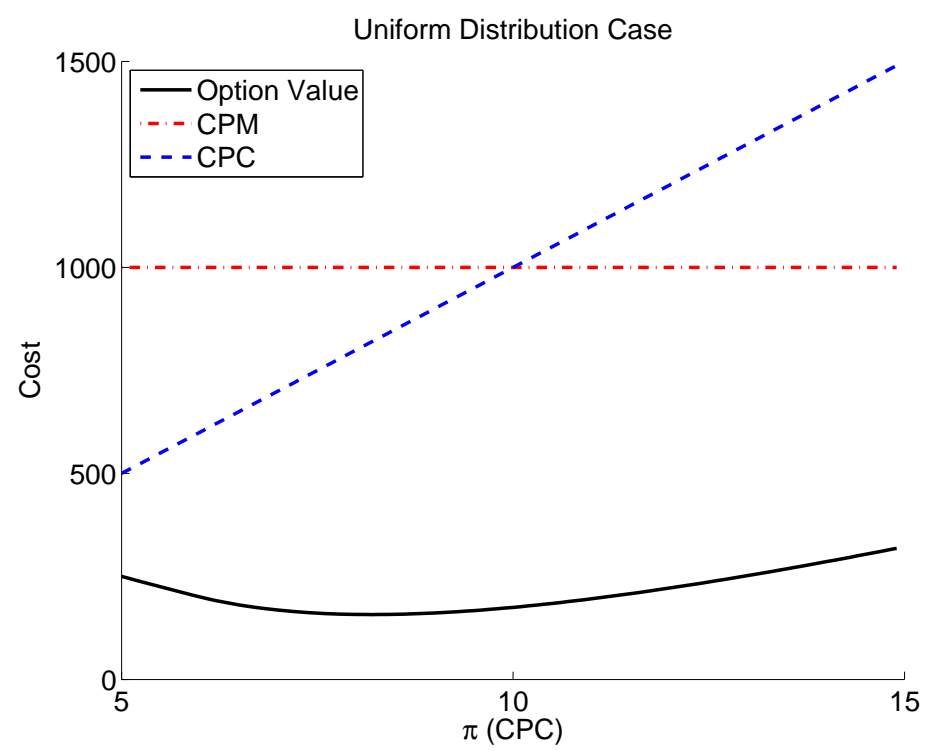

Figure 10: Option values with varying $\pi$ (CPC)

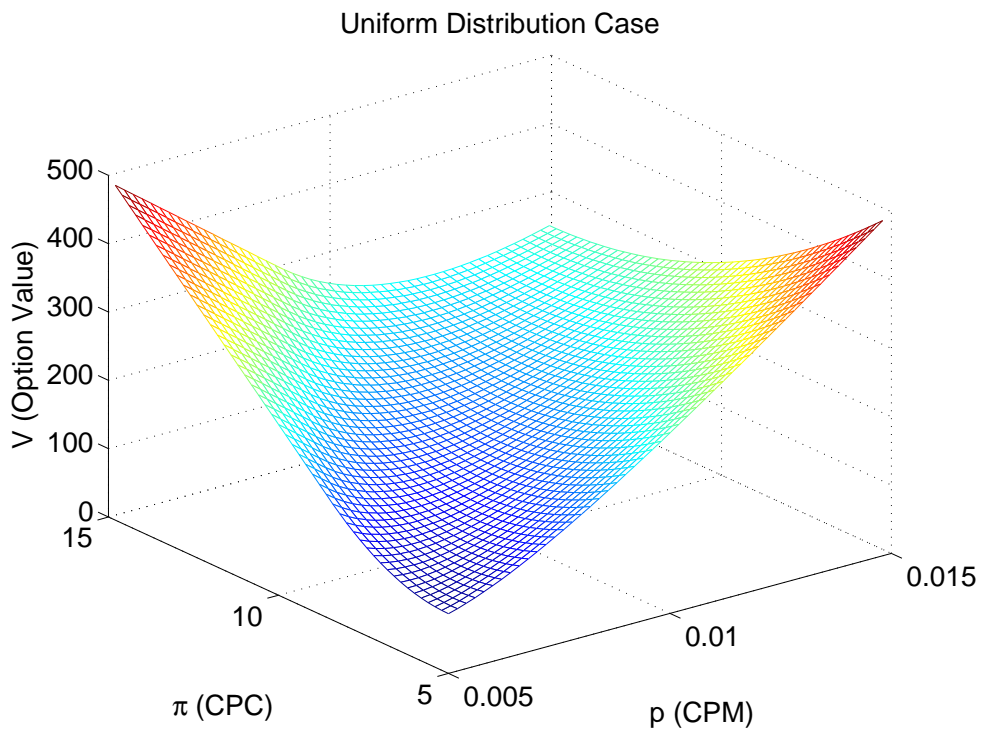

Figure 11: Option values with varying $p(\mathrm{CPM})$ and $\pi(\mathrm{CPC})$ 
For the case of $c_{1} \leq p / \pi \leq c_{2}$, we have the option value as:

$V$

$$
\begin{aligned}
& =\frac{1}{2}\left(a \pi n \frac{c_{1}+c_{2}}{2}+b p n\right)-\frac{n \pi}{c_{2}-c_{1}}\left[\frac{(p / \pi)^{2}}{2}-\frac{\left(c_{1}\right)^{2}}{2}\right]-n p\left(1-\frac{p / \pi-c_{1}}{c_{2}-c_{1}}\right) \\
& =\frac{1}{2}(a \pi n \bar{c}+b p n)-\frac{n \pi}{2 \delta}\left[\frac{(p / \pi)^{2}}{2}-\frac{(\bar{c}-\delta)^{2}}{2}\right]-n p\left(1-\frac{p / \pi-\bar{c}+\delta}{2 \delta}\right)
\end{aligned}
$$

Therefore

$$
\begin{aligned}
\frac{\partial V}{\partial(\operatorname{Var})} & =\frac{\partial V}{\partial \delta} \cdot \frac{\partial \delta}{\partial(\operatorname{Var})} \\
& =\frac{\partial V}{\partial \delta} \cdot \frac{3}{2 \delta} \\
& =-\frac{n}{4 \delta^{2} \pi} \frac{3}{2 \delta}(p-\pi \bar{c}+\pi \delta)(p-\pi \bar{c}-\pi \delta)
\end{aligned}
$$

However from the condition $c_{1} \leq p / \pi \leq c_{2}$, we have

$$
\bar{c}-\delta \leq p / \pi \leq \bar{c}+\delta
$$

or

$$
\pi \bar{c}-\pi \delta \leq p \leq \pi \bar{c}+\pi \delta
$$

Therefore, we obtain

$$
\frac{\partial V}{\partial(\operatorname{Var})} \geq 0
$$

for all Var. This result is quite consistent with an intuitive belief: the higher risk (variance) we face, the bigger protection cost (option value) we need.

However, for general cases, the derivative $\frac{\partial V}{\partial(\operatorname{Var})}$ cannot be expressed in a closed-form solution. Therefore, we study the relationship numerically. For both the gamma and uniform distributions we considered, we compute the option values changing the variance of CTR, while keeping the mean value of CTR constant. For the gamma distribution, $\operatorname{Gamma}(k, \theta)$, the variance is given by $\operatorname{Var}=k \theta^{2}$. The result is presented in Figure 12. The option value increases monotonically as the variance of CTR increases. 


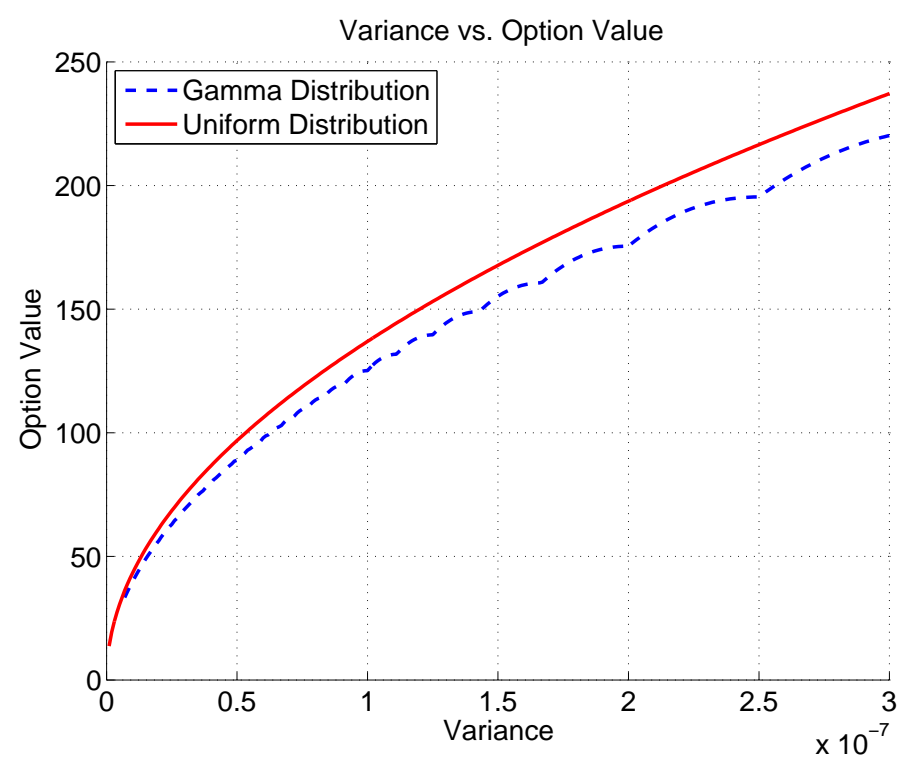

Figure 12: Option values with varying variances for gamma and uniform distributions

\section{Conclusion}

In this paper, we proposed a new type of option contract for online advertising, and evaluated its properties. In Internet advertising, the costper-impression (CPM) and the cost-per-click (CPC) are widely used as the basis for contracting. However, because of uncertainty in click-through rates (CTR), one contract may cost more than the other to an advertiser or bring more revenue to a publisher and vice versa. For this reason, the selection of contract can be a crucial decision making issue to both publishers and advertisers. In this context, publishers and advertisers are willing to hedge the uncertainty of future advertising cost, as a means to achieve higher revenue. Thus, this research suggests an option to pay the minimum of CPM and CPC fees as a way to avoid the ambiguity of the contract type choice. The advertiser can avoid future high costs by purchasing the option in advance, while the publisher can generate additional revenue by selling the option. We derived an appropriate pricing rule for such options by solving for a game-theoretic equilibrium involving two players.

From the proposed model, we have shown that the option contract is beneficial in the aspects of (i) contract feasibility, (ii) publisher's utility and 
(iii) advertiser's regret. First, our result shows that the option contract will increase the chance of a contract between the advertiser and publisher being made, because an option contract can be agreed to even for a relatively lower CTR. Also, this result shows that a publisher can have more potential demand (advertisers) using the option contract, when we consider the whole market. When CTR is predicted to be very low for an advertiser's campaign, the advertiser prefers CPC, while the publisher prefers CPM. Therefore, sometimes no agreement is possible only with CPM and CPC. However, an option contract can be used as a good tool to mitigate the conflicting interests of both parties, because it has a lower threshold of CTR. Consequently, the publisher can contract with more advertisers even for a low CTR. Second, another result showed that a publisher can make the most profits by introducing an option contract, when the difference between the expected and realized CTRs is small. This can be a great advantage to the publisher, but also suggests that the publisher should try to accurately estimate the CTR. Finally, we showed that the advertiser can expect a stable level of regret regardless of CTR. As we know, CPM and CPC might lead to losses in some cases such as too low CTR and the existence of click fraud. In this environment, contract selection could be a source of regret. However, an advertiser who chooses an option contract does not have to worry about this environmental issue or extreme scenarios.

In practice, option contracts can be used in a number of different ways. An advertiser can avoid confusion in online advertising contract selection (risk hedging). Although display advertisements are usually contracted based on CPM in current practice as they have been in traditional media, there are many debates about how online advertisement contracts should be made. The main source of this confusion is CTR. Should an advertisement campaign receive many clicks, the advertiser will prefer to pay based on CPM. On the other hand, when an advertisement campaign receives a small number of clicks, the advertiser will prefer to pay based on CPC. However, the publisher will have exactly the opposite preferences. Using the option contract we proposed in this paper, we can eliminate a source of confusion to a certain degree. Moreover, using an option contract offers a good opportunity to a publisher who hopes to make more profit. More importantly, online advertisement is basically beneficial to both parties, because both can earn profits from advertisement. But, due to their conflicting interests, they could lose this opportunity. However, our proposed option contract can play a role in mitigating the conflict and support the simultaneous interests of 
an advertiser and a publisher who are worried about inappropriate contract selection and profitability.

This research has a number of limitations, since we did not consider some contract factors to keep our analysis relatively simple. To make the model more realistic, we could consider, for example, duration of contract, advertising format (still image, digital video and rich media), and so on. More importantly, we could consider how a banner is displayed. It can be displayed as fixed in a page for a day or a week, or can be displayed with other banners mixed for a longer period. In the former case, for a day or a week, it will receive more attention, but predicting CTR will be harder.

This research also can be extended to consider each player's risk attitude. Some players are more afraid of uncertainty than others. This attitude will increase or decrease the option value. Another extension is to address the possibility of asymmetric information. Usually, a publisher may have more information than an advertiser for the number of incoming customers. This can result in advantage for the publisher and in this situation the option price will change also. Thus, addressing the potential for a moral hazard problem will be an interesting topic to analyze further using a principal agent model and a game-theoretic approach.

Although our focus has been on the direct negotiation between an advertiser and a publisher, we may extend our approach to consider a negotiation between advertisers and the network agency that connects advertisers with multiple publishers. In practice, many publishers maintain two contracting channels: one for direct negotiation with advertisers as studied in this paper, and the other with agencies. To apply our approach to the second case, we need to resolve the issue of measuring click-through rates, because an advertisement through agencies is often displayed by multiple numbers of web sites. A simple way to do this is to use the distribution of the average click-through rates of the many web sites.

\section{Acknowledgement}

The authors thank the editor Robert Kauffman, the associate editor, and two anonymous reviewers for their invaluable comments and detailed suggestions. They are also grateful to Ikkjin Ahn for helpful discussions. 


\section{References}

Adler, M., Gibbons, P., and Matias, Y. Scheduling space-sharing for Internet advertising. Journal of Scheduling, 5, 2, 2002, 103-119.

Aggarwal, G., Feldman, J., and Muthukrishnan, S. Bidding to the top: VCG and equilibria of position-based auctions. In T. Erlebach and C. Kaklamanis (eds.), Lecture Notes in Computer Science, Springer-Verlag, Berlin Heidelberg, volume 4368. 2007, 15-28.

American Press Institute. Newspaper economic action plan. Technical report, American Press Institute, Reston, Virginia, May 2009.

Amiri, A., and Menon, S. Scheduling web banner advertisements with multiple display frequencies. IEEE Transactions on Systems, Man, and Cybernetics, Part A: Systems and Humans, 36, 2, 2006, 245-251.

Athey, S., and Ellison, G. Position auctions with consumer search. NBER Working Paper No. w15253., 2009. Available at SSRN: http://ssrn.com/abstract=1454986. Last accessed on April 23, 2010.

Campbell, J. Real options analysis of the timing of IS investment decisions. Information \& Management, 39, 5, 2002, 337-344.

Dawande, M., Kumar, S., and Sriskandarajah, C. Performance bounds of algorithms for scheduling advertisements on a web page. Journal of Scheduling, 6, 4, 2003, 373-394.

DoubleClick. 2008 year-in-review benchmarks. Research report, DoubleClick, New York, June 2009. Available at: http://www.doubleclick.com/insight/pdfs/dclk_2008benchmarks_0906.pdf. Last accessed on April 23, 2010.

Feng, J., Bhargava, H., and Pennock, D. Implementing sponsored search in web search engines: Computational evaluation of alternative mechanisms. INFORMS Journal on Computing, 19, 1, 2007, 137-148.

Fjell, K. Online advertising: Pay-per-view versus pay-per-click - A comment. Journal of Revenue and Pricing Management, 8, 2009, 200-206. 
Fridgeirsdottir, K., and Najafi-Asadolahi, S. The optimal pay-per-click pricing of display advertising in advertising networks: A queuing systems approach. Working Paper, London Business School, London, U.K., 2009a.

Fridgeirsdottir, K., and Najafi-Asadolahi, S. Revenue management for online advertising using advertising networks. Working Paper, London Business School, London, U.K., 2009b.

Gallego, G., Kou, S. G., and Phillips, R. Revenue management of callable products. Management Science, 54, 3, 2008, 550-564.

Gallego, G., and Phillips, R. Revenue management of flexible products. Manufacturing \& Service Operations Management, 6, 4, 2004, 321-337.

Hoffman, D., and Novak, D. P. How to acquire customers on the web. Harvard Business Review, 78, 3, 2000, 179-188.

Interactive Advertising Bureau. IAB Internet Advertising Revenue Report. Technical report, Interactive Advertising Bureau, New York, U.S.A., 2008. Available at: http://www.iab.net/insights_research/530422/1357. Last accessed on April 23, 2010.

Kumar, R. A framework for assessing the business value of information technology infrastructures. Journal of Management Information Systems, 21, 2, 2004, 11-32.

Kumar, S., and Sethi, S. Dynamic pricing and advertising for web content providers. European Journal of Operational Research, 197, 3, 2009, 924944.

Kwon, C. Single-period balancing of pay-per-click and pay-per-view online display advertisements. Journal of Revenue and Pricing Management, in press.

Lahaie, S., Pennock, D., Saberi, A., and Vohra, R. Sponsored search auctions. In N. Nisan, T. Roughgarden, E. Tardos, and V. V. Vazirani (eds.), Algorithmic Game Theory, Cambridge University Press, Cambridge, U.K. 2007, 699-716.

Levin, Y., McGill, J., and Nediak, M. Price guarantees in dynamic pricing and revenue management. Operations Research, 55, 1, 2007, 75-97. 
Liu, D., Chen, J., and Whinston, A. Ex ante information and the design of keyword auctions. Information Systems Research, in press.

Mangani, A. Online advertising: Pay-per-view versus pay-per-click. Journal of Revenue and Pricing Management, 2, 4, 2004, 295-302.

Nagarajan, M., and Sošić, G. Game-theoretic analysis of cooperation among supply chain agents: Review and extensions. European Journal of Operational Research, 187, 3, 2008, 719-745.

Nash, J., Jr. The bargaining problem. Econometrica, 18, 2, 1950, 155-162.

Robinson, H., Wysocka, A., and Hand, C. Internet advertising effectiveness: the effect of design on click-through rates for banner ads. International Journal of Advertising, 26, 4, 2007, 527-541.

Roels, G., and Fridgeirsdottir, K. Dynamic revenue management for online display advertising. Journal of Revenue and Pricing Management, 8, 2009, 467-468.

Roth, A. E. Axiomatic Models of Bargaining, volume 170 of Lecture Notes in Economics and Mathematical Systems. Springer-Verlag, 1979. Available at: http://kuznets.fas.harvard.edu/ aroth/Axiomatic_Models_of_Bargaining.pdf. Last accessed on April 23, 2010.

Varian, H. Position auctions. International Journal of Industrial Organization, 25, 6, 2007, 1163-1178.

Wilmott, P., Dewynne, J., and Howison, S. Option Pricing: Mathematical Models and Computation. Oxford Financial Press, Oxford, U.K., 1993. 